\title{
Coagulase Negative Staphylococcus Causes Catheter Associated Bacteriemia in a Patient with Esophagus Adenocarcinome
}

\author{
A. Copca-Álvarez, Miguel Ángel Cabrera-Suarez, Gerardo Pulido-Reyes, Julia Alcoba-Florez, \\ Manuel Morales, Sebastián Mendez-Alvarez* \\ Carretera del Rosario, Hospital Universitario Nuestra Señora de Candelaria, Santa Cruz de Tenerife, Spain. \\ Email: *sebastianmendez@funcis.org
}

Received July $26^{\text {th }}$, 2012; revised August $30^{\text {th }}$, 2012; accepted September $9^{\text {th }}, 2012$

\begin{abstract}
We report a case of a man suffering esophagus adenocarcinome who acquired catheter associated bacteriemia caused by a coagulase negative Staphylococcus. This CoNS was sensible to linezolid, teicoplanine, vancomycin and rifampicin. This information was relevant for antibiotherapy planning. The patient was successfully treated with teicoplanin together with the catheter exchange. In conclusion, infections should be treated with adequate doses and duration of antibiotics together with catheter exchange. Pre-emptive measurements in the cancer patient and establishing the most adequate treatment are imperative for obtaining good results.
\end{abstract}

Keywords: Coagulase Negative Staphylococcus; Catheter; Bacteriemia; Teicoplanin; Esophagus Adenocarcinome

\section{Introduction}

Primary prevention is the first step any society can take to reduce the incidence of cancer, with positive effects on other noncommunicable diseases. Addressing certain lifestyle choices (tobacco cessation, decreased alcohol consumption, healthy diet and physical activity) has been shown to improve population health and reduce cancer deaths. Environmental and occupational risk factors should mainly be tackled by traditional health protection activities. At a policy level, population-based mass screening programmes, which have been proven to reduce cancer mortality substantially, must be clearly, distinguished from opportunistic screening, for which there is insufficient evidence of population benefit. Screening programmes must be based on solid evidence. Once cancer has been diagnosed, truly integrated care should be the goal of the health system. The care pathways between diagnosis, treatment (including chemotherapy, radiation and surgery), psychological support, palliative care and rehabilitation need to be well organized in order to optimize outcomes (including quality of life) for all patients (European Observatory on Health Systems and Policies, www.euro.who.int). Definetively, fight against cancer is a hard way but coordination between all health related areas makes it much easier. Accordingly then, weakness

\footnotetext{
"Corresponding author.
}

against infections agents could not happen in the chain against cancer. Infections in people who have cancer or are getting cancer treatment can be more serious than those in other people. They can also be harder to treat. If you have cancer, it is important to find infections early and treat them quickly, before they get worse and spread. Cancer itself can increase the risk of getting a serious infection. So can certain types of cancer treatment. Once the cancer is gone and treatment is finished, the risk of infection usually goes back to a normal level. For most people, high-level risk for serious infection only lasts for a limited time (American Cancer Society, www.cancer.org). Therefore, pre-emptive measurements in the cancer patient and an adequate microbiologic characterization of the germ causing infection in order to establish the most adequate treatment are imperative for obtaining good results.

\section{Case Report}

A 57-year-old man was admitted on December 2011 to the emergency department of the Hospital Universitario Nuestra Señora de Candelaria (HUNSC) showing clinical signs of progressive Dysphagia, with an associated lost of $9 \mathrm{~kg}$ in last three weeks. He was examined by endoscopy which permitted visualizing esophagus neoformation. His anamnesis included, not known allergies, ex-smoker (20 - 30 cigarettes per day), exalcohol drinker 
(50 gr. per day), and cold thyroids node with secondary hypothyroidism. Treatment against this last disorder was levotiroxin $75 \mathrm{mg}$ per day. His main body characteristics were: Weight 83 Kg, Height 182 cm, PS0; Rhythmic hearth noises without pathologic aggregates; Soft abdomen, without any organ hyperplasia; Normal up and down extremities; Neurological examination: normal. His familial anamnesis included a brother died with head and neck neoplasia, a second brother with neoplasia in ENL, a sister with gynaecologic neoplasia, father with cerebral neoplasia and mother with hepatic neoplasia.

In 2012, analyses of the esophagus neoformation were continued what permitted to conclude that the patient was affected by an Esophagus Adeno Carcinome T4N2M0. At that moment, Chemotherapeutic Treatement was initiated; First line therapy included CDDP $\left(100 \mathrm{mg} / \mathrm{m}^{2}\right)$, $200 \mathrm{mg}$ per day, 1 day; 5-F (1000 mg/m²), $2.000 \mathrm{mg}$ per day during 5 days. This treatment was applied every 21 days by 2 cicles. After this first line chemotherapy, Distal Esophagus Adeno carcinome T4N2M1 in progression was observed. Then second line therapy was commenced; Second line therapy comprised Taxotere in monotherapy. Additionally, in March $19^{\text {th }}$, 2012, Radiochemotherapy over tumoral macroscopic zone was initiated; FD: 200 cGy, per day, until a total of 5 doses per week, TD: 4000 cGy.

In May of the same year, the patient manifested a burst of Febrile Neutropeny Grade IV. In May 21 ${ }^{\text {st }}$ Blood analysis results were $\mathrm{Hb} 8.4 \mathrm{~g} / \mathrm{dl}$, Hct 25.2, MCV $77 \mathrm{fl}$, platelet count. $178,000 / \mathrm{mm}^{3}, 600$ leukocytes $/ \mathrm{mm}^{3}$ (200 neutrophils $/ \mathrm{mm}^{3}, 100$ monocytes $/ \mathrm{mm}^{3}$, 300 lymphocytes/ $\mathrm{mm}^{3}$ ) glucose 148 , urea 30 , creatinine 0.7 , Sodium 132, Potassium 3.2. Calcium 8, GOT/GPT 59/78. Arterial blood gas: pH 7.49, pO2 82, pCO2 36. Sat 97\% baseline. Fever and cells count putatively suggested an infection. Chest X-ray exhibted regular Mediastinum with not infiltrated mass or images. Catheter in the right anterior chest was exchanged. Then, blood samples and a section of the upper part of the catheter were sent to the Microbiology Service of the Hospital. There, two hemocultures recovered from a piece of the catheter and from systemic blood, respectively, after $48 \mathrm{~h}$ were positive for a Coagulase Negative Staphylococcus spp. Antibiotic susceptibilities determined by the Vitek2 system showed that the staphylococci was susceptible to Linezolide, Teicoplanine, Vancomycin, Rifampicin. Intravenous treatment with Teicoplanine was then prescribed and started at initial dose of $20 \mathrm{mg} / \mathrm{kg}$; following doses depending on its blood levels and maintained for 21. After this period, the patient showed clinical improvement and to date is stable with an AV fistula as permanent vascular access.

\section{Discussion}

Among staphylococci, Staphylococcus aureus is the most virulent species and the most evil pathogen, but the incidence of infections caused by Coagulase-negative staphylococci (CoNS) has increased throughout the world [1]. CoNS are usually multidrug-resistant including cotrimoxazole, erythromycin, quinolones, clindamycin, tetracycline and chloramphenicol [2]. Glycopeptide antibiotics have been to date considered the drugs of choice for treatment [3]. Furthermore, various virulence factors are responsible for the symptoms and severity of infections caused by Staphylococcus spp. Among them are staphylococcal enterotoxins (SEs) and toxic shock syndrome toxin-1 (TSST-1). Some reports indicate that TSST-1 and staphylococcal enterotoxins are also produced by CoNS [4,5]. Moreover, catheter-related infection in cancer patients constitutes an important health-care problem with major financial implications. During the last years a better understanding of the pathogenesis of catheter-related infections and the interaction between microorganisms and catheter surfaces has grown up. CoNS have been established as a group of bacteria exhibiting different virulence factors that favors biofilm development. The influence of biofilm formation in catheter-related infections has been established. The development of biofilm by the colonizing microbes permits attachment of the organisms to the vascular access device and confers resistance to antibiotics and host defense mechanisms [6]. In this case report we found a CoNS sensible to linezolid, teicoplanine, vancomycin and rifampicin causing a catheter associated bacteriemia in a patient with esophagus adenocarcinome. This information was relevant for antibiotherapy planning. Treatment with teicoplanine solved the infection. In conlusion, we want to show that the rapid institution of a highly efficacious treatment is essential. Infections should be treated with adequate doses and duration of antibiotics together with catheter exchange. Pre-emptive measurements in the cancer patient and an adequate microbiologic characterization of the germ causing infection in order to establish the most adequate treatment are imperative for obtaining good results [5].

\section{Acknowledgements}

This work was partially supported by grants FIS06/0002 and FIS10/00125 from INSTITUTO DE SALUD CARLOSIII (Spanish Health Ministry) to S.M.-A.

\section{REFERENCES}

[1] H. Sakai, G. W. Procop, N. Kobayashi, D. Togawa, D. A. Wilson, L. Borden, V. Krebs and T. W. Bauer, "Simultaneous Detection of Staphylococcus aureus and Coagulase-Negative Staphylococci in Positive Blood Cultures by Real-Time PCR with Two Fluorescence Resonance Energy Transfer Probe Sets,” Journal of Clinical Micro- 
biology,Vol. 42, No. 12, 2004, pp. 5739-5744.

doi:10.1128/JCM.42.12.5739-5744.2004

[2] R. A. Proctor, "Coagulase-Negative Staphylococcal Infections: A Diagnostic and Therapeutic Challenge," Clinical Infectious Diseases, Vol. 31, No. 1, 2000, pp. 31-33. doi:10.1086/313894

[3] S. Natoli, C. Fontana, M. Favaro, A. Bergamini, G. P. Testore, S. Minelli, M. C. Bossa, M. Casapulla, G. Broglio, A. Beltrame, L. Cudillo, R. Cerretti and F. Leonardis, "Characterization of Coagulase-Negative Staphylococcal Isolates from Blood with Reduced Susceptibility to Glycopeptides and Therapeutic Options," BMC Infectious Diseases, Vol. 4, No. 1, 2009, p. 83.

doi:10.1186/1471-2334-9-83
[4] N. Gaebler and M. Ribeiro de Souza, "Staphylococcal Enterotoxins: Molecular Aspects and Detection Methods,” Journal of Public Health and Epidemiology, Vol. 2, No. 3, 2010, pp. 29-42.

[5] M. Macía, J. Donate, J. Alcoba-Florez and S. MendezAlvarez, "Daptomycin, Methicillin Resistant Staphylococcus hominis Catheter-Related Bacteriemia in a Hemodialysis Patient," Open Journal in Medical Microbiology, 2012(in press).

[6] M. Morales, S. Méndez-Alvarez, J. V. Martín-López, C. Marrero and C. O. Freytes, "Biofilm: the Microbial 'Bunker' for Intravascular Catheter-Related Infection,” Support Care Cancer, Vol. 12, No. 10, 2004, pp. 701-707. doi:10.1007/s00520-004-0630-5 